\title{
Ermis: a middleware for bridging data collection and data processing in IoT streaming applications
}

\author{
Stefanos Peros, Wouter Joosen, Danny Hughes \\ imec-DistriNet, KU Leuven \\ 3001, Leuven, Belgium \\ Email: firstname.lastname@kuleuven.be
}

\begin{abstract}
Modern streaming analytics platforms, such as Apache Flink, can receive, process, and act on real-time streaming data from various sources to take immediate action as events occur in the real world. Combined with the emergence of the Internet of Things (IoT), these platforms have revolutionized many application domains by deriving strategic insights from incoming sensor data. For instance, manufacturers can retrofit production lines with sensors, enabling them to rapidly detect and correct problems by analyzing incoming sensor data in realtime. However, when sensors generate data too slowly, the application produces incorrect results due to insufficient information. Conversely, generating data too fast rapidly decreases sensor battery lifetime and wastes storage resources. Consequently, the data generation rate of the IoT infrastructure must match the data processing rate of the application(s) at all times, which is a challenging task due to the several facets of IoT dynamism: sensor mobility, sensor failure, changing application data requirements, varying message inter-generation and transmission delay. Currently, application developers are required to manually configure sensor sampling periods to match these rates; a timeconsuming process that is inefficient, if not ineffective, due to the dynamism of the IoT infrastructure. In this paper, we tackle this problem by introducing Ermis, a novel middleware that runs on the gateway and automatically adapts sensor sampling periods at runtime to match application requirements, without relying on any previous knowledge about the underlying IoT infrastructure. To the best of our knowledge, this is the first solution that can ensure the presence of sufficient data for the IoT applications under various sources of dynamism, while minimizing unnecessary transmissions to extend sensor battery lifetime. Extensive evaluation on real-time data from a physical testbed demonstrated a $\sim 34 \%$ increase in the expected battery lifetime, while consistently ensuring data sufficiency, compared to state-of-the-art.
\end{abstract}

Index Terms-internet of things, data collection, data processing, adaptive sampling, middleware, streaming applications

\section{INTRODUCTION}

The emergence of the Internet of Things (IoT) has created immense opportunities for companies to gain market advantage over their competitors through sensor data collection and processing. For many applications, the sooner sensor data is processed, the more meaningful the insights that can be extracted from it, enabling timely reactions to events as they occur in the real world. In pursuit of that goal, companies often rely on modern streaming analytics tools, such as Apache Flink [1] and Azure Stream Analytics [2], to analyze and process a wide range of sensor data. These platforms offer an intuitive way to process and combine individual sensor data streams through sliding window queries [3]-[9] which periodically process recent events.

On the one hand, when sensors are transmitting too slowly, the quality of the produced result is negatively impacted due to insufficient events in sliding windows [10]-[13]. On the other hand, when sensors are transmitting too fast, redundant transmissions waste both application storage resources and sensor energy, which is scarce for resource-constrained batterypowered devices [10], [14], [15]. Thus, it is crucial to bridge the gap between data collection needs and the capabilities of data processing to ensure sustainable IoT applications.

In practice, sensors in industrial networks are typically configured with preset sampling intervals [10], [16]. However, whereas application developers are experts with respect to the data processing needs of their application, they have little knowledge over the data collection process in the IoT infrastructure, which is often managed by the infrastructure providers [17]. This differentiation of stakeholders, together with the inherent platform, network and application dynamism of the IoT, poses unique challenges that render data collection at preset time intervals inefficient. In particular, sensor transmissions often deviate from the configured sampling period (message inter-generation delay), due to the lack of network synchronization and the typical absence of a real-time clock. Furthermore, network latency also varies over time, causing packets to arrive at their destination(s) at a non-constant rate [11], [16]. Finally, the amount of data required by the application can be linked to the sensor location, which can change at runtime due to sensor mobility that is typical in many IoT infrastructures.

A number of previous studies have investigated and proposed adaptive sampling approaches to overcome the limitations of predetermined sampling periods. These approaches focus on adapting sensor sampling rate with respect to the rate of change of the sampling object, varying from complex distributed algorithms [18]-[20] to more centralized gatewaylevel solutions [10]. While these techniques can improve the sampling accuracy of the measured physical property, they do not consider application data requirements and thus offer no guarantees that sufficient data will be present at the time of application query execution to produce correct results, as our evaluation shows. That is because the sampling object's rate of change is not the only factor to affect how much data applications require for processing at runtime, as described in 
our use case.

In this paper, we propose Ermis, a novel middleware that runs on the gateway and tackles the problem of matching sensor data collection and processing for IoT streaming analytics in the presence of platform, network and application dynamism. To the best of our knowledge, Ermis is the first solution that guarantees compliance with application data requirements, while minimizing redundant sensor sampling and transmissions. Ermis achieves this by continuously monitoring the delay and state of the underlying IoT infrastructure, along with application data requirements, to automatically adapt sensor sampling periods. We evaluate Ermis on top of a realworld testbed for various platform, network and application dynamism conditions. The results show that Ermis consistently satisfies application queries, unlike state-of-the-art approaches, while minimizing data redundancy and thus extending sensor battery lifetime by $\sim 34 \%$.

The remainder of the paper is structured as follows: Section II specifies the model and problem statement, and illustrates the problem by means of an example industrial use-case. Section III gives an overview of the related work. Section IV describes our proposed adaptive sampling strategy and the architecture of the middleware. Section V presents the implementation details of the approach and the testbed. Section VI discusses the evaluation setup and results. Finally, Section VII concludes the paper and provides directions for future work.

\section{BACKGROUND}

\section{A. Model \& Problem Statement}

Streaming applications are typically modelled using three key concepts: event, stream, and query. An event $\boldsymbol{e}$ corresponds to a single measurement that is sampled and transmitted in a message by a sensor to the gateway. It is modelled as a tuple of four attributes: $\langle s r c, g t, a t, v\rangle$, corresponding to the sensor, generation timestamp, arrival timestamp, and measurement value respectively. A sensor stream $s$ models a sensor that generates events at a configurable sampling period $p$, and is characterized by set of key-value pairs named tags, e.g. "location: Production Floor". The difference between consecutive event generation timestamps is equal to the sum of the sampling period $p$ and the inter-generation delay igd, whereas the transmission delay trd is equal to the difference between the event arrival at and generation timestamp gt. Both igd and trd vary over time in wireless sensor networks and for each sensor. Ermis does not rely on prior knowledge regarding these delays, but instead monitors the underlying infrastructure continuously to estimate their values.

A query $\boldsymbol{q}$ has a length $l$, which determines the number of events in its window $w$ that it processes every period $p$ time units. Sensor streams inserted in a query's window when their tags match, i.e. by streams with at least the same tags as the query. When multiple sensors are sampling a common object, their measurements can be combined to satisfy the query length, which we refer to as an aggregate query. Delaysensitive queries filter out late event arrivals due to delay by only processing events that are generated after the last time that the query executed.

The problem at hand consists of two objectives: maximizing the sensor stream sampling period, or equivalently minimizing its energy consumption, while ensuring that when each query executes, the number of events in its window is at least equal to its length. We define the optimization problem that Ermis aims to solve as follows:

Problem 1: (ENERGY OPTIMIZATION). Given a sensor stream $\boldsymbol{s}$ and a query $\boldsymbol{q}$ with window $\boldsymbol{w}$ and length $\boldsymbol{l}$, the Energy Optimization aims to find a sampling period $\boldsymbol{p}$ such that: $\max p$

$$
\text { subject to: } \operatorname{COUNT}(w) \geq l
$$

\section{B. Motivating Example}

The motivating example in this paper is an industrial use case on the monitoring of an airbag manufacturing division of a Fortune 500 car-safety equipment producer. The manufacturing plant consists of the storage floor and the assembly floor, the latter containing multiple production lines, and operates under a two-shift schedule, commonly used by European car manufacturers [21].

Each production line is equipped with sensors that measure the item processing rate, indicating how efficiently the line produces airbags, and the quality of the produced airbags to detect defects. When the number of defect airbags across the production lines exceeds a threshold, e.g. as regulated by ISO 26262 [22], production must halt and all produced airbags of the current batch must be discarded. Workers drive transportation vehicles, which are equipped with localization sensors, to deliver materials from the storage to the assembly floor. Additionally, each floor has sensors to monitor the ambient temperature. Together, all these sensors form a multihop wireless mesh network, over which they periodically transmit measurements to the gateway under varying delay.

The monitoring application collects sensor data from the gateway, which it processes to update a visual display that is monitored by a human operator in real-time. More concretely, the display is divided into four areas, one per sensor type, and each area corresponds to a sliding window query whose period and length are specified by the operator. The update frequency of each window, i.e. query period, must be respected at all times (hard deadline) to maintain a clear link between the displayed results and their occurrence in real-time. Furthermore, sufficient sensor data must be present whenever a window is refreshed to ensure that correct results are displayed. The next section discusses related work and derives the middleware requirements.

\section{RELATED WORK}

To date, several studies have investigated adaptive sampling techniques that improve energy consumption and data accuracy in wireless sensor networks. The authors in [23] propose ASAP, an adaptive sampling approach that clusters sensors into sampler and non-sampler nodes. The latter sensors do not transmit sensor data to preserve energy; instead, ASAP 
uses probabilistic models to predict their values, which can be subject to prediction errors. Jain et al. [20] suggest an adaptive sampling approach that consists of a client and a server module. The client runs on the sensor, enabling it to autonomously adjust its sampling period by means of a Kalman Filter, based on the size of the prediction error. Kho et al. [19] investigate the benefits of a decentralized algorithm, where each sensor is responsible for finding its optimal sampling frequency based on the value of the produced information. However, a common limitation of the aforementioned approaches is the modification to the sensor source code and the underlying networking protocol, which limits their applicability to existing real-world deployments. This is because it is often difficult, if not impossible, to alter the code of each sensor in large-scale deployments, let alone the routing protocol of the gateway. Additionally, these sensors typically have limited energy and computational resources, rendering it difficult to run such complex algorithms.

To overcome these limitations, recent research has focused on gateway-level adaptive sampling solutions. The authors of [10] propose a data-driven approach that automatically adapts the sensor sampling period based on the characteristics of change in the sampling object. For that purpose, the authors estimate the change trend of the sampling object using a linear median jitter sum, increasing (decreasing) the sampling period as the sum increases (decreases). However, the approach does not take into account application data requirements, nor the varying delay, which can cause sensor data to arrive too late at the applications. This also holds for other state-of-theart techniques that have been compared in a recent survey [15] on adaptive sampling approaches for the IoT. Thus, a mismatch between application queries and data collection is still likely to occur, resulting in either insufficient data or excessive transmissions.

\section{Requirements}

From our motivating example and the related work, we identify four requirements for the adaptive sampling middleware:

- R1: Support the registration/deletion of application sliding window queries in a technology agnostic fashion. This ensures integration with present and future data streaming platforms that support sliding window based primitives.

- R2: Ensure that the condition of the ENERGY OPTIMIZATION problem, defined in Section II-A, is satisfied for each registered query at all times.

- R3: Automatically discover sensor data streams in the underlying IoT infrastructure, but also detect and react to changes in their characteristics. This ensures that the middleware can operate in a plug-and-play fashion on top of existing and future IoT infrastructure(s), removing the burden of manual user (re)configuration and the need for prior infrastructure knowledge.

- R4: Forward incoming sensor data only to the intended sliding window queries to minimize the communication overhead between the middleware and the streaming platforms that host the applications.

\section{ARCHITECTURE}

\section{A. Adaptive Sampling Strategy}

The adaptive sampling strategy that is used by Ermis can be modelled as the transition between an Idle State and an Optimization State. In the former, sensor sampling periods are not being modified: it is the default state. Changes to sensor streams and application queries can trigger a transition to the Optimization State, in which the sampling periods for each relevant stream are recomputed to ensure sufficient data (requirement R2), before returning to the Idle State after reconfiguration until the next trigger occurs. Based on our motivating example, we identify the following eight triggers. Sensors can (1) join and (2) leave the network, and their properties can change at runtime, including (3) their location and (4) delay. Queries can also be (5) added, (6) removed, and their (7) length and (8) period can be reconfigured at runtime.

In the Optimization State, Algorithm 1 is used to compute the optimal sampling period for each stream that is related to the trigger. The algorithm uses Algorithm 2 to find the largest period (slowest transmission rate) that satisfies all queries that match the stream's tags (Lines 7-13). If there are no queries associated with the given stream, it returns an arbitrarily large sampling period (Lines 4-5) to preserve energy.

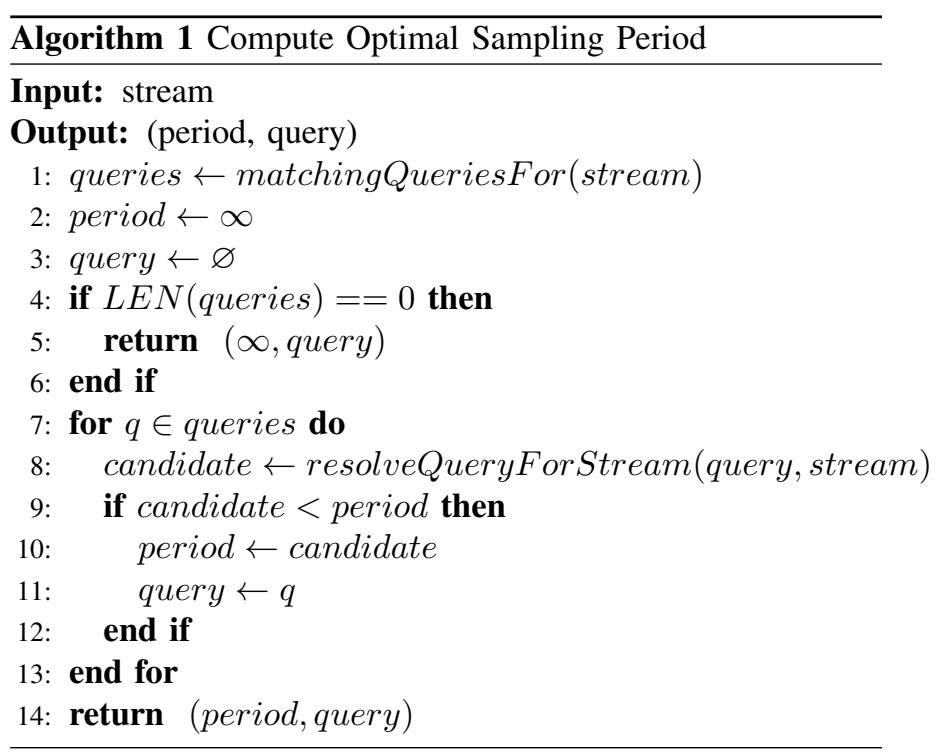

Given a stream and a query, Algorithm 2 returns the largest sampling period that satisfies the query's length, resulting in the least amount of transmissions. If the query is delaysensitive, the transmission delay of the stream is taken into account (Lines 2-6). Note that the stream's inter-generation delay is taken into account regardless, as it impacts event generation time (Line 3, 23, 32). For an aggregate query, all associated streams that contribute to the satisfaction of that query's length are considered (Lines 8-26). For streams whose period is defined by queries with stricter data requirements, the algorithm subtracts their number of transmitted events from the query length (Line 27). If no additional events are required to satisfy the query, then an arbitrarily large sampling period is 
returned (Lines 29-30). Otherwise, the algorithm distributes the workload among the number of remaining streams (Line 28) that can be reconfigured by the given query (Line 31 ).

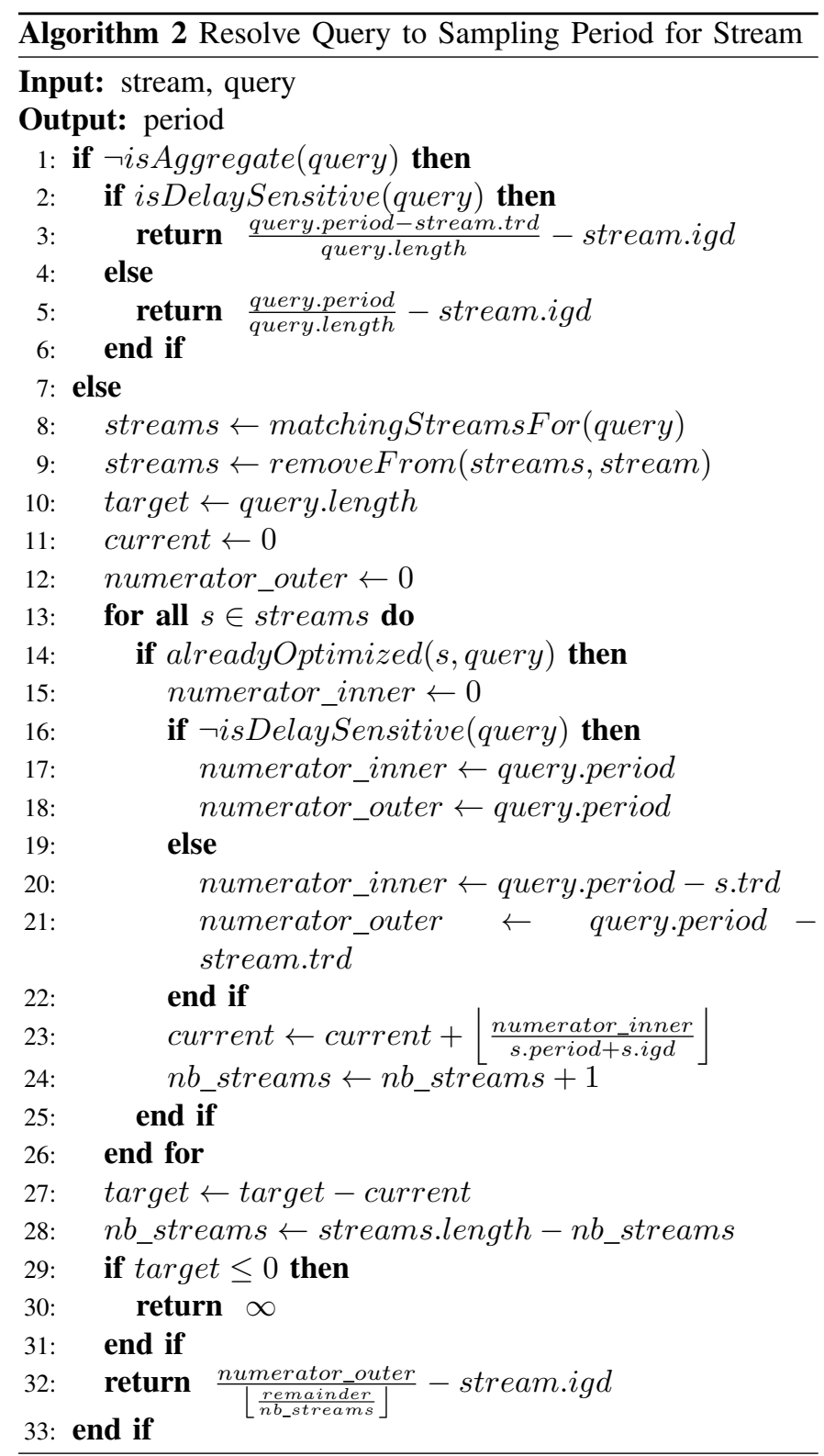

Note that not all recomputed sampling periods must necessarily result in a reconfiguration, as this would cause significant overhead. In many applications, including our use case, saving energy should not come at the cost of insufficient sensor data, which is why we take a conservative approach: always re-configure the sensor if it should transmit faster. However, when it can transmit slower, the sensor is only reconfigured if it would decrease the number of transmission by at least $10 \%$; a threshold that can be configured by the user.

\section{B. Components}

Fig. 1 shows an architectural overview of the middleware, including the components and external interfaces, which is

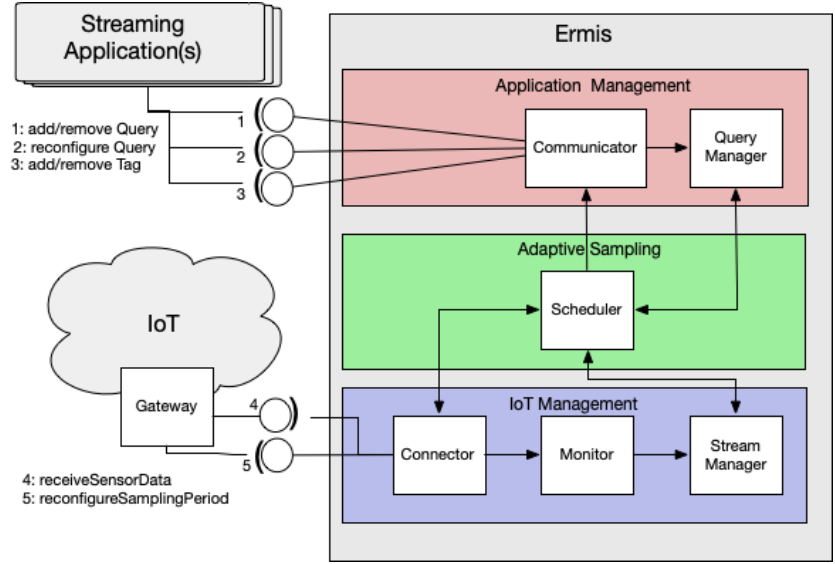

Fig. 1: Ermis Component Diagram.

divided into three layers based on their respective roles and responsibilities.

The Application Management layer consists of two components: Communicator and Query Manager. The Communicator is responsible for providing an Application Programming Interface (API) to the streaming applications (requirement R1) to create, delete and reconfigure queries. It also forwards incoming sensor data from the Scheduler to the relevant queries (requirement $\mathbf{R 4}$ ). In practice, the Communicator can be distributed over multiple physical nodes to scale with the number of streaming applications. The Query Manager is responsible for maintaining an overview of the registered queries, and notifies the Scheduler when a query change occurs to trigger a transition to the $O p$ timizization State, where sampling periods are re-computed (requirement R2).

The Adaptive Sampling layer implements the adaptive sampling strategy, discussed in Section IV-A. The Scheduler is responsible for (a) matching queries with streams based on their tags, and (b) solving the optimization problem stated in Section II-A by means of the aforementioned algorithms. This component obtains an overview of the registered queries and discovered streams from the Query Manager and Stream Manager respectively. Furthermore, it forwards incoming sensor data to the Communicator (requirement R4), and issues sampling period re-configurations in the IoT infrastructure through the Connector.

The IoT Management layer consists of three components. The Connector is responsible for communicating with the gateway(s) of the underlying IoT infrastructure(s); it listens for incoming sensor data from each gateway, which it parses and forwards to the Scheduler and Monitor. In practice, the Connector can be distributed over multiple physical nodes for scalability, to cope with a large number of IoT infrastructures. The Monitor is responsible for requirement R3: it checks the source of incoming sensor messages to discover new streams, detects changes in the sensor's location by checking the message metadata, and estimates the intergeneration and transmission delay for each stream by ana- 
lyzing the generation and arrival timestamps, as discussed in Section V-A. The Stream Manager maintains an overview of the discovered streams and notifies the Scheduler when a stream change occurs to trigger a transition to the Optimizization State (requirement R2).

\section{IMPLEMENTATION}

\section{A. Middleware}

Ermis is implemented in Node.js, which uses the asynchronous event-driven runtime of JavaScript. As a result, the middleware uses minimal resources since Node.js sleeps when there is no work to be done, making it possible to run Ermis on the gateway $\left(500 \mathrm{MHz}\right.$ Intel Atom ${ }^{\mathrm{TM}}$ Processor, 1GB RAM), which has important benefits in terms of latecy [10], [17]. The complete code-base is documented and will be made available as open-source at the time of publication.

The Communicator uses Representational State Transfer (REST) to implement the API that applications can use to register, remove and reconfigure queries. Furhermore, the Communicator serves a websocket to which it forwards sensor data to the relevant queries (requirement R4). The IoT gateway provides a minimal REST API that enables sampling period reconfiguration and a websocket, both of which are used by the Connector to reconfigure sensors and receive incoming sensor messages respectively.

Since our middleware does not depend on a priori knowledge of the underlying IoT infrastructure, it relies on monitoring to estimate the delay for each sensor stream. More concretely, the Monitor component uses worst case estimates for the inter-generation and transmission delays, similar to TCP's Retransmission TimeOut Timer [24], based on the experimental observation that over $99 \%$ of possible values are within four standard deviations of the mean. These estimates are computed using an exponentially smoothed slow-moving mean $(\alpha=0.875)$ and fast-moving variance $(\alpha=0.75)$ :

$$
\begin{aligned}
& t r d_{\text {est }}=\operatorname{tr} d_{\text {mean }}+4 * t r d_{\text {var }} \\
& i g d_{\text {est }}=i g d_{\text {mean }}+4 * i g d_{\text {var }}
\end{aligned}
$$

Worst-case estimates are chosen to improve robustness against insufficient sensor data, in the absence of prior infrastructure knowledge, to ensure that the condition of the optimization problem formulated in Section II-A is satisfied.

\section{B. IoT Network}

The IoT network is implemented using SmartMesh IP, a state-of-the-art industrial IoT network technology. Devices are connected wirelessly, forming a multi-hop mesh topology that can adapt itself at runtime to guarantee high network reliability and ultra low-power; properties that are desirable for our use case. The mesh topology, along with the ongoing selfadaptation, result in varying delay and thus non-deterministic message arrival times. The gateway supports acknowledgements, and thus sensor messages can be re-transmitted until they are successfully delivered. SmartMeshIP uses a TimeSynchronized Channel Hopping (TSCH) MAC protocol that organizes time in slots, which are allocated to sensors in the network, enabling them to know in advance when to turn the radio on or off.

In total, there are 32 physical devices in the testbed: 1 VersaSense edge gateway (Model M01), 11 VersaSense wireless devices (Model P02), and 20 SmartMesh IP motes (DC9003A-B), deployed indoors over an area of 90 square meters. Each VersaSense wireless device is equipped with up to four peripherals (plug-and-play sensors), resulting in a total of 34 individual sensor data streams. The SmartMesh IP motes do not sense the environment: they instead forward incoming messages across the network to enable a widespread deployment with a large number of hops. Furthermore, our deployment is configured with the following network parameters $^{1}$ : basebw $=100 \mathrm{~ms}$, bwmult $=900 \%$, corresponding to the minimum period between transmissions, and the bandwidth over-provisioning.

\section{Vi. EVAluation}

\section{A. Setup}

We extensively evaluate Ermis in the presence of various sources of platform, network, and application dynamism, which do not only apply to our use case but are also generally present in any IoT context. All experiments are performed on top of the real-world testbed that was described in the previous section. We evaluate against two approaches: static sampling, which is commonly used in practice, and data-driven adaptive sampling, which is proposed by the authors in [10]. In the former approach, sensor sampling periods are set to their largest sampling periods that satisfy the queries at the start of the experiment, and do not change at runtime without manual reconfiguration. The latter is a state-of-the-art approach, which is proposed by the authors in [10] and discussed in Section III, where sensor sampling periods are reconfigured at runtime based on the change in value of the measured property. For comparison, we use three metrics: Query Violation, Data Redundancy and Energy Consumption. Query violation is the percentage of query executions with insufficient sensor data. Data redundancy is the percentage of additional sensor data that was collected, compared to the query length. Both of these metrics are computed as moving averages over a window of the past 100 query executions. As we are interested in the total sensor energy consumption (mAh) over a long period of time, since the gateway is mains powered, we use the built-in Coulomb Counter ${ }^{2}$ of each VersaSense wireless device.

\section{B. Experiments}

a) Performance with Dynamic Application Requirements: As described in our use case, the query length can vary at runtime, as specified by the operator. The first experiment tests how Ermis copes with increasing application requirements in terms of the query length. This experiment consists of

\footnotetext{
${ }^{1}$ https://www.analog.com/media/en/technical-documentation/userguides/smartmesh_ip_user_s_guide.pdf

${ }^{2}$ https://www.analog.com/media/en/technical-documentation/datasheets/3335f.pdf
} 
three application queries, one for each approach, with identical periods (20 minutes) and increasing lengths of 20, 40, and 60 events. Each application query matches a different VersaSense Wireless Device that is equipped with four peripherals. The experiment lasted six days, involving over 1200 query executions and over 34000 sensor messages. The results are shown in Fig. 2a, where each vertical line corresponds to a query length reconfiguration. Unlike Ermis, both the data driven and static approaches violate the query after reconfiguration (Subplot 1), which is why their data redundancy drops to $0 \%$ (Subplot 2). Instead, Ermis sufficiently decreases sensor sampling periods (Subplot 3), taking into account the new query lengths and the observed delay, which results in an overall data redundancy of less than $10 \%$.

The second experiment tests how Ermis copes with decreasing application requirements in terms of the query period. This experiment consists of three application queries, one for each approach, with identical lengths (20 events) and increasing periods of 10, 15, and 20 minutes. The experiment lasted four days, involving over 1150 query executions and over 30000 sensor data. The results are shown in Fig. 2b, where each vertical line corresponds to a query period reconfiguration. As queries require the same number of events over a longer period of time, none of the approaches violate the query length. However, Ermis reacts to the change in query period by reconfiguring sensors to transmit slower, maintaining a data redundancy of $7-8 \%$ throughout the experiment; up to two orders of magnitude less than the other two approaches.

b) Performance with Sensor Mobility: As described in our use case, sensors attached on vehicles can change locations as these move between the storage and the assembly floor. The goal of this experiment is to test how Ermis copes with sensor mobility. For each approach, there are two queries $q_{\text {storage }}$ and $q_{\text {assembly }}$ with identical periods (10 minutes) and lengths (20 events). Initially, there are five sensor streams for $q_{\text {storage }}$, and four for $q_{\text {assembly }}$. At runtime, a vehicle changes floors, resulting in four streams for $q_{\text {storage }}$ and five for $q_{\text {assembly }}$ instead. This experiment lasted three days, involving over 2500 query executions and 50000 sensor data.

Fig. 2c and Fig. 2d show the results for $q_{\text {storage }}$ and $q_{\text {assembly }}$ respectively, as the mobile sensor moves from the storage to the assembly floor, which is indicated by the vertical lines. On the one hand, Ermis decreases the sampling period of the remaining sensors on the storage floor, taking into account the observed delay as always. On the other hand, it increases the sampling period of the sensors on the assembly floor, as there are now more sensors in that location. Consequently, Ermis results in query satisfaction and a low data redundancy ( $<10 \%)$ for both $q_{\text {storage }}$ and $q_{\text {assembly }}$, in contrast to the other two approaches.

c) Performance with Sensor Failure: This experiment focuses on how Ermis copes with sensor failures, which are common in any wireless IoT infrastructure. There are in total three queries, one per approach, with identical length (20 events) and period (20 minutes). Each query matches with eight sensor streams, corresponding to two VersaSense Wire- less Devices, each equipped with four peripherals. Throughout this experiment, we introduce sensor failures by removing a peripheral from each of the VersaSense wireless devices, thus reducing the number of available sensor data streams by $25 \%$ in each step. The experiment lasted seven days days, and involves over 1500 query executions and over 16000 sensor data.

The results are shown in Fig. 2e, where the timestamps of sensor failure correspond to the vertical lines. Ermis detects each sensor failure and re-configures the remaining peripherals to decrease their sampling period, ensuring that the query data requirements remain satisfied while maintaining a $\sim 5 \%$ data redundancy. The other two approaches, however, violate their respective queries as the number of sensor streams decreases.

d) Performance w.r.t Energy Consumption: In this experiment, we measure and compare the performance of each approach in terms of energy consumption, based on the twoshift operation schedule of the manufacturing plant in our use case. More concretely, queries execute fast during peak production hours (every 15 seconds) and slower otherwise (every 3 minutes). In total, we've measured and compared the energy consumption across the entire testbed over the course of 30 days.

Fig. 2f illustrates the impact of the energy consumption on the expected lifetime of the devices, based on their battery capacity (3000mAh). The lines correspond to the mean energy consumption of each approach over time, whereas the shaded areas correspond to one standard deviation from the mean. Ermis keeps data redundancy low while satisfying the query by re-configuring sensors based on the operation schedule. On average, this extends battery lifetime by $\sim 34 \%$ (from 12 to 16 months) compared to the other approaches, thus reducing the frequency at which batteries need to be replaced.

\section{Discussion}

The evaluation has covered several dynamic scenarios, which are based on our use-case, but can be easily generalized to most IoT infrastructures. Indeed, sensor data streams can become unavailable at any time due to sensor failures, as a result of battery depletion, physical damage or a software crash. Also in the context of smart cities, sensors are attached to public transportation vehicles, changing locations as they drive around the city. Finally, many activity monitoring applications, e.g. patient monitoring, adjust the sliding window query length at runtime as a function of the observed activity, changing the amount of required sensor data. Our evaluation has shown the limitations of state-of-the-art adaptive sampling approaches, which result in either insufficient or abundant sensor data. The poor performance of the static sampling approach is expected, as it does not adapt sensor sampling periods at runtime. However, the results also show the limitations of using a data-driven adaptive sampling approach [10], from which we conclude that adapting sensor sampling periods solely on how much the value of the measured property changes is inadequate for satisfying application sliding window queries in the presence of IoT dynamism. Instead, Ermis considers query requirements 
as constraints to be satisfied, and relies on monitoring the underlying state of the infrastructure, which makes it also applicable to other IoT networks. The results have shown that our approach leads to consistent query satisfaction, and at most $10 \%$ data redundancy, thus tackling the problem stated in Section II-A.

\section{CONCLUSION}

The IoT has revolutionized data collection from physical processes, commonly through sliding window queries in streaming analytics and applications. Sufficient data needs to be present upon each sliding window query execution to produce correct results, whereas the abundance of sensor data results in wasted storage resources and shortened sensor battery lifetimes. Thus, there is a clear need for automated and fine-grained control over data collection and processing, which is challenging due to the inherent IoT dynamism.

In this paper, we propose Ermis, a novel middleware that supports streaming application developers by guaranteeing that sliding window query data requirements are satisfied, while minimizing data redundancy and consequently energy consumption in the IoT infrastructure. Our approach is implemented in Node.js and can run on the gateway, which is mains powered. Ermis has been extensively evaluated and compared against state-of-the-art approaches under various sources of platform, application and network dynamism, on top of a real-world testbed. The results show that Ermis not only consistently satisfies sliding window query data requirements, but that it also extends battery lifetime by $\sim 34 \%$ compared to state-of-the-art.

A natural progression of our work is to further reduce data redundancy, which is currently capped at $10 \%$. This is a natural consequence of using worst case estimates for the delay, since we have designed our approach without a priori knolwedge of the underlying IoT infrastructure. Instead, more accurate delay models can be obtained by studying in greater depth the delay characteristics of leading IoT network technologies (e.g. BLE, LoRaWAN), which in turn would improve the accuracy of the adaptation, thus further extending battery lifetime.

\section{REFERENCES}

[1] P. Carbone, A. Katsifodimos, S. Ewen, V. Markl, S. Haridi, and K. Tzoumas, "Apache flink: Stream and batch processing in a single engine," Bulletin of the IEEE Computer Society Technical Committee on Data Engineering, vol. 36, no. 4, 2015.

[2] A. Basak, K. Venkataraman, R. Murphy, and M. Singh, Stream Analytics with Microsoft Azure: Real-time data processing for quick insights using Azure Stream Analytics. Packt Publishing Ltd, 2017.

[3] J. Li, K. Tufte, V. Shkapenyuk, V. Papadimos, T. Johnson, and D. Maier "Out-of-order processing: a new architecture for high-performance stream systems," PVLDB, vol. 1, pp. 274-288, 012008.

[4] P. Carbone, A. Katsifodimos, S. Ewen, V. Markl, S. Haridi, and K. Tzoumas, "Apache flink ${ }^{\mathrm{TM}}$ : Stream and batch processing in a single engine," IEEE Data Engineering Bulletin, vol. 38, 012015.

[5] Y. Ji, J. Sun, A. Nica, Z. Jerzak, G. Hackenbroich, and C. Fetzer, "Quality-driven disorder handling for m-way sliding window stream joins," 2016 IEEE 32nd International Conference on Data Engineering (ICDE), May 2016. [Online]. Available: http://dx.doi.org/10.1109/ICDE.2016.7498265

[6] K. Kenda, B. Kazic, E. Novak, and D. Mladenić, "Streaming data fusion for the internet of things," Sensors, vol. 19, p. 1955, 042019.
[7] U. Srivastava and J. Widom, "Flexible time management in data stream systems," in Proceedings of the Twenty-Third ACM SIGMOD-SIGACTSIGART Symposium on Principles of Database Systems, ser. PODS '04. New York, NY, USA: Association for Computing Machinery, 2004, p. 263-274. [Online]. Available: https://doi.org/10.1145/1055558.1055596

[8] N. Rivetti, Y. Busnel, and A. Mostéfaoui, "Efficiently summarizing data streams over sliding windows," in 2015 IEEE 14th International Symposium on Network Computing and Applications, 2015, pp. 151158.

[9] Y. Ji, J. Sun, A. Nica, Z. Jerzak, G. Hackenbroich, and C. Fetzer, "Quality-driven disorder handling for m-way sliding window stream joins," in 2016 IEEE 32nd International Conference on Data Engineering (ICDE), 2016, pp. 493-504.

[10] P. Lou, L. Shi, X. Zhang, Z. Xiao, and J. Yan, "A data-driven adaptive sampling method based on edge computing," Sensors, vol. 20, p. 2174 , 042020.

[11] N. Rivetti, N. Zacheilas, A. Gal, and V. Kalogeraki, "Probabilistic management of late arrival of events," 06 2018, pp. 52-63.

[12] Z. Liao, X. Lu, T. Yang, and H. Wang, "Missing data imputation: a fuzzy k-means clustering algorithm over sliding window," in 2009 Sixth International Conference on Fuzzy Systems and Knowledge Discovery, vol. 3. IEEE, 2009, pp. 133-137.

[13] O. Farhat, H. Bindra, and K. Daudjee, "Leaving stragglers at the window: Low-latency stream sampling with accuracy guarantees," in Proceedings of the 14th ACM International Conference on Distributed and Event-Based Systems, ser. DEBS '20. New York, NY, USA: Association for Computing Machinery, 2020, p. 15-26. [Online]. Available: https://doi.org/10.1145/3401025.3401732

[14] S. Tarannum et al., "Energy conservation challenges in wireless sensor networks: A comprehensive study," Wireless Sensor Network, vol. 2, no. 06, p. 483,2010

[15] D. Giouroukis, A. Dadiani, J. Traub, S. Zeuch, and V. Markl, "A survey of adaptive sampling and filtering algorithms for the internet of things," in Proceedings of the 14th ACM International Conference on Distributed and Event-Based Systems, ser. DEBS '20. New York, NY, USA: Association for Computing Machinery, 2020, p. 27-38. [Online]. Available: https://doi.org/10.1145/3401025.3403777

[16] S. Peros, S. Delbruel, S. Michiels, W. Joosen, and D. Hughes, "Khronos: Middleware for simplified time management in cps," in Proceedings of the 13th ACM International Conference on Distributed and Event-Based Systems, ser. DEBS '19. New York, NY, USA: Association for Computing Machinery, 2019, p. 127-138. [Online]. Available: https://doi.org/10.1145/3328905.3329507

[17] S. Akkermans, S. Peros, N. Small, W. Joosen, and D. Hughes, "Supporting iot application middleware on edge and cloud infrastructures." in ZEUS, 2018, pp. 40-46.

[18] J. Ploennigs, V. Vasyutynskyy, and K. Kabitzsch, "Comparative study of energy-efficient sampling approaches for wireless control networks," IEEE Transactions on Industrial Informatics, vol. 6, no. 3, pp. 416-424, 2010.

[19] J. Kho, A. Rogers, and N. R. Jennings, "Decentralised adaptive sampling of wireless sensor networks," 2007

[20] A. Jain and E. Y. Chang, "Adaptive sampling for sensor networks," in Proceeedings of the 1st international workshop on Data management for sensor networks: in conjunction with VLDB 2004, 2004, pp. 10-16.

[21] S. Lehndorff, "Working time and operating hours in the european automotive industry," Institut für Arbeit und Technik Gelsenkirchen, 2000.

[22] "ISO 26262-7 - Road vehicles - Functional safety - Part 7: Production and operation," https://www.en-standard.eu/iso-26262-7-road-vehiclesfunctional-safety

-part-7-production-operation-service-and-decommissioning/, Accessed: 2019-12-12.

[23] B. Gedik, L. Liu, and S. Y. Philip, "Asap: An adaptive sampling approach to data collection in sensor networks," IEEE Transactions on Parallel and distributed systems, vol. 18, no. 12, pp. 1766-1783, 2007.

[24] A. S. Tanenbaum, D. Wetherall et al., Computer networks. Prentice hall, 1996. 


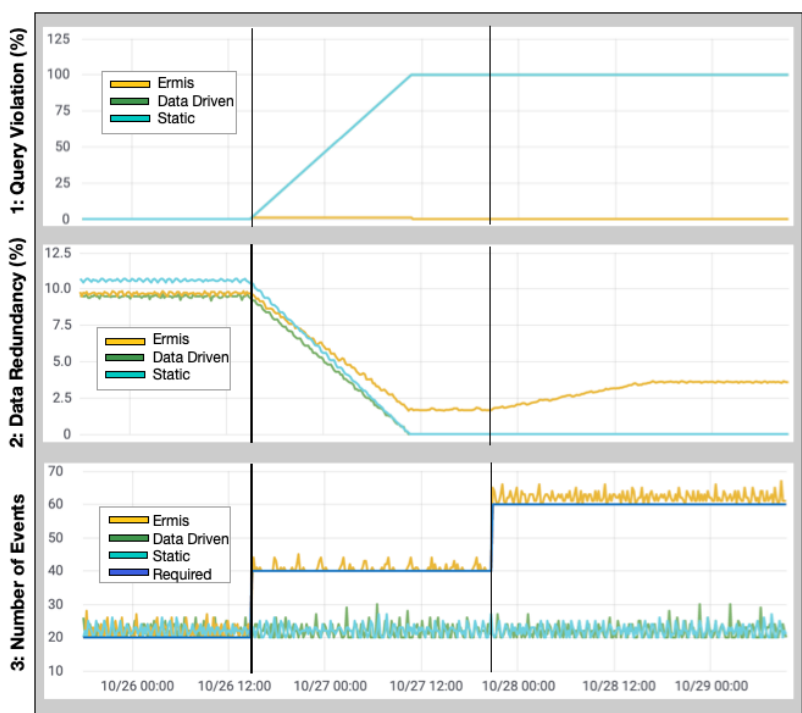

Time (MM/dd HH:mm)

(a)

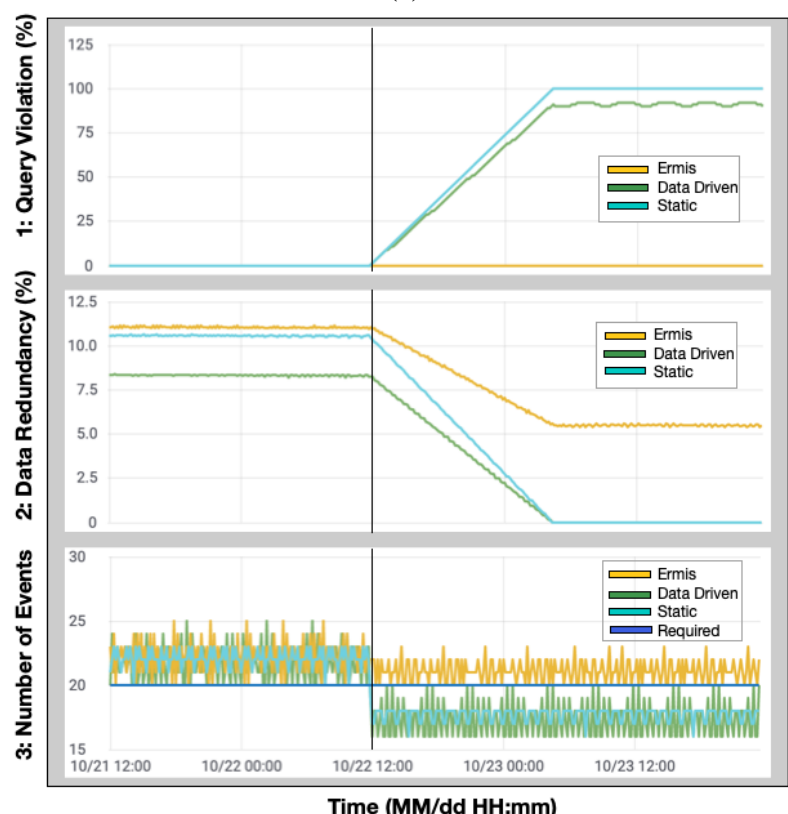

(c)

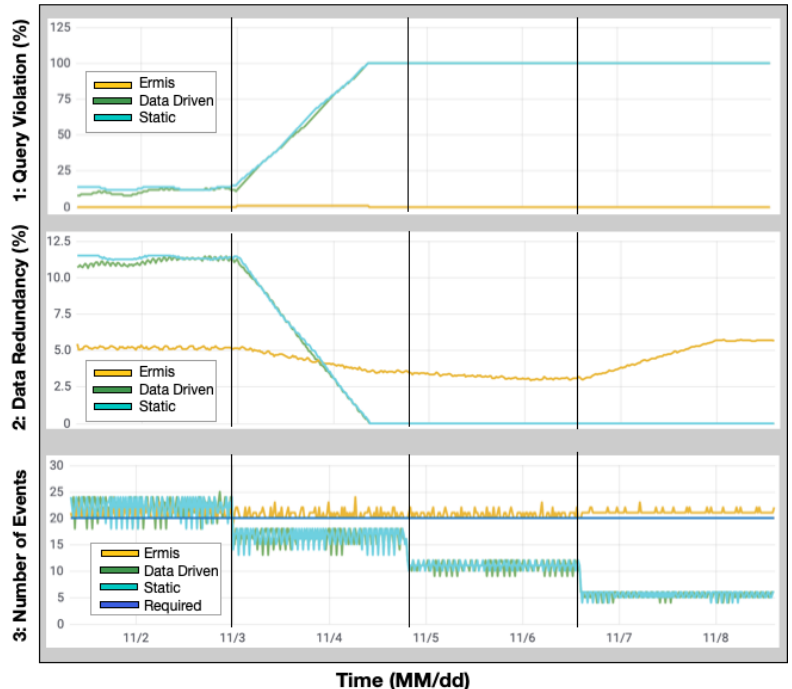

(e)

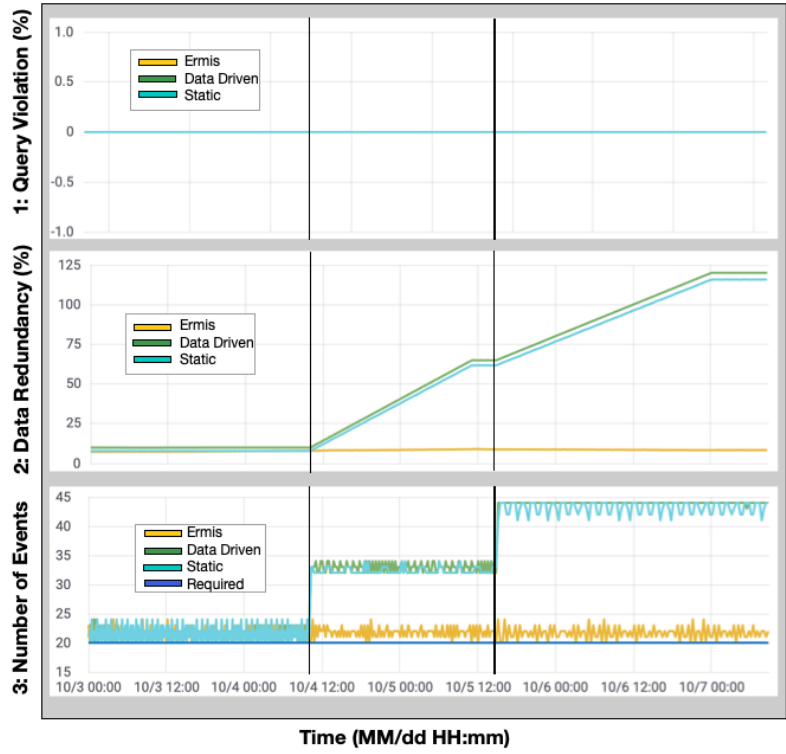

(b)

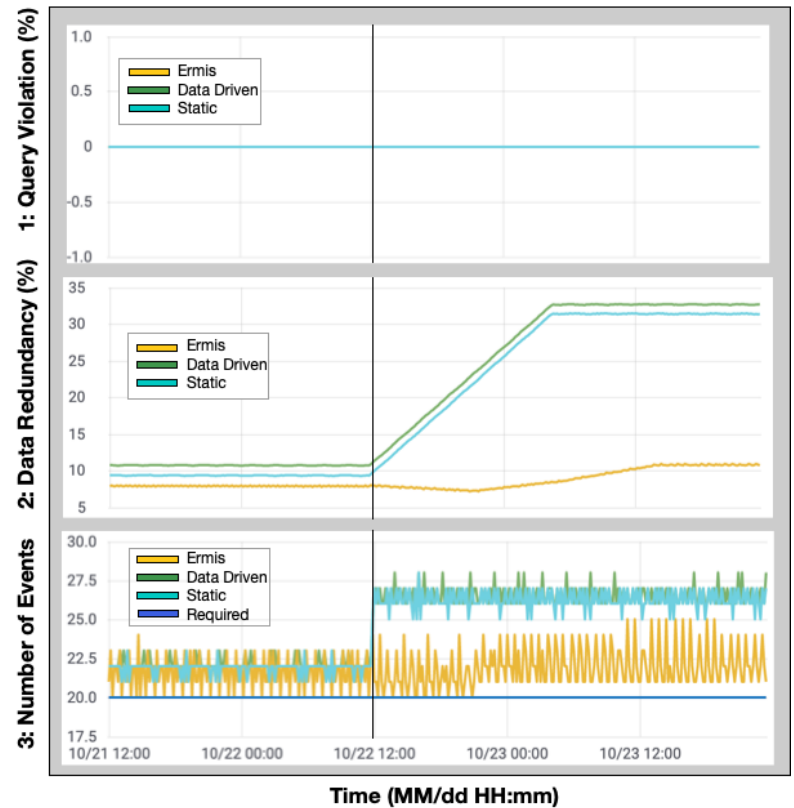

(d)

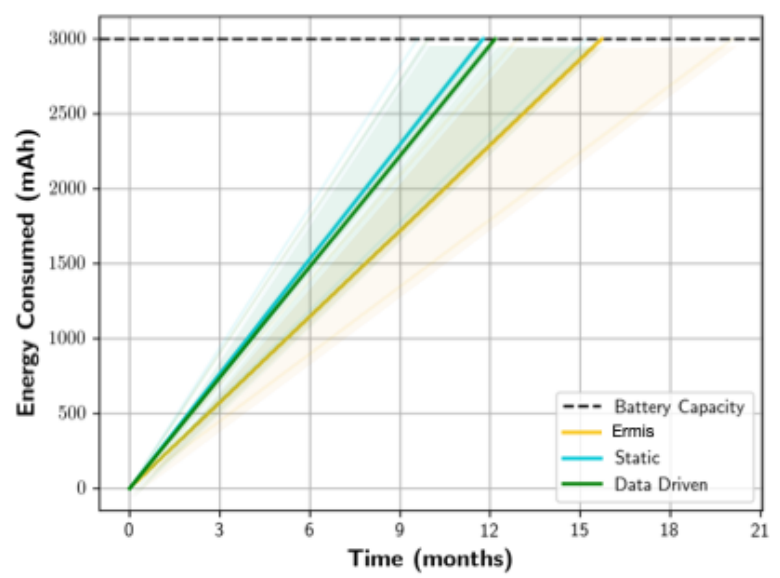

(f)

Fig. 2: Evaluation results for various dynamism scenarios: query length (a), query frequency (b), sensor mobility (c,d), sensor failure (e); and impact on energy consumption (f) over a long period of time. 